\title{
Single scattering analysis of speckle correlation
}

\author{
Chen Bar $^{\mathrm{a}}$ Marina Alterman $^{\mathrm{a}}$ Ioannis Gkioulekas ${ }^{\mathrm{b}}$ Anat Levin ${ }^{\mathrm{a}}$ \\ ${ }^{a}$ Department of EE, Technion, Israel. ${ }^{b}$ Robotics Institute, CMU, USA.
}

\begin{abstract}
We propose a closed-form expression for memory effect speckle correlations, enriching our understanding of their unique properties. The model considers only the single-scattering component of light, yet offers a good approximation even in thicker volumes. (C) 2021 The Author(s)
\end{abstract}

\section{Introduction}

Coherent light scattering through participating media, such as biological tissue, creates noise-like images known as speckle. Despite their seemingly random nature, speckle patterns have strong statistical correlation properties such as the memory effect (ME), which means that speckle patterns produced under nearby illumination conditions are correlated shifted versions of each other. ME has been successively applied in many computational imaging systems, significantly expanding our ability to image through random media. Despite their large potential, speckle statistics are not well understood, as accurate closed-form formulas characterizing them are lacking.

Analytical expressions for speckle statistics such as the Fokker-Planck or the diffusion approximation [4] rely on simplifying assumptions, making them valid for relatively thick scattering volumes. In such volumes ME correlations are very weak and their practical applicability is limited [1].

In this abstract we present a recent alternative closed-form model we proposed in [3], relying on the first Born approximation to light scattering, or equivalently, considering only the single scattering component of the light [2]. We show that this model holds even in materials whose optical depth is much larger than one, providing surprisingly good approximation to speckle correlations in regimes where diffusion models are completely invalid.

\section{Modeling speckle statistics}

Let us denote by $u^{\hat{\mathbf{i}}}(\hat{\mathbf{v}})$ the speckle field scattered from a material illuminated by an incoming plane wave at direction $\hat{\mathbf{i}}$ toward viewing direction $\hat{\mathbf{v}}$. We measure speckle correlation as

$$
\mathcal{C}\left(\hat{\mathbf{i}}^{1}, \hat{\mathbf{i}}^{2}, \hat{\mathbf{v}}^{1}, \hat{\mathbf{v}}^{2}\right)=E\left[u^{\hat{\mathbf{i}}^{1}}\left(\hat{\mathbf{v}}^{1}\right) \cdot u^{\hat{\mathbf{i}}^{2}}\left(\hat{\mathbf{v}}^{2}\right)^{*}\right]
$$

where $u^{\hat{\mathbf{i}}^{1}}\left(\hat{\mathbf{v}}^{1}\right), u^{\hat{\mathbf{i}}^{2}}\left(\hat{\mathbf{v}}^{2}\right)$ are two fields scattered by the same material under two different illuminations, and the expectation is taken with respect to the fields scattered by multiple slabs from the same type of material (e.g. multiple tissue slices of the same type and thickness).

While classical approaches in optics calculated the fields by solving the wave equation, [2] calculates it as an integral over all light paths, illustrated in Fig. 1(a). Consider a path $\overrightarrow{\mathbf{x}}=\mathbf{o}_{0} \rightarrow \ldots \rightarrow \mathbf{o}_{B+1}$ with $\mathbf{o}_{0}=\hat{\mathbf{i}}, \mathbf{o}_{B+1}=\hat{\mathbf{v}}$, where $B=0, \ldots, \infty$. It contributes a complex throughput $\xi(\overrightarrow{\mathbf{x}})$ encapsulating the amplitude attenuation along the path and the phase corresponding to its length. A pair of paths has a complex contribution

$$
\left.c_{\hat{\mathbf{v}}^{1}, \hat{\mathbf{v}}^{2}}^{\hat{\mathbf{i}}^{1}} \hat{\hat{\mathbf{x}}}^{2}, \overrightarrow{\mathbf{x}}^{1}\right) \propto \xi\left(\overrightarrow{\mathbf{x}}^{1}\right) \xi\left(\overrightarrow{\mathbf{x}}^{2}\right)^{*} .
$$

The expected correlation of Eq. (1) was shown in [2] to be equivalent to the integral over all pairwise path contributions in Eq. (2). However, for most path pairs Eq. (2) contributes a complex number with rather random phase, and the average of such numbers is zero. Here, we suggest that many of the longer path pairs contribute random phases and decorrelate quickly. We argue that in fact, most correlation arrives from single scattering paths of the form $\overrightarrow{\mathbf{x}}^{1}=\hat{\mathbf{i}}^{1} \rightarrow \mathbf{o} \rightarrow \hat{\mathbf{v}}^{1}, \overrightarrow{\mathbf{x}}^{2}=\hat{\mathbf{i}}^{2} \rightarrow \mathbf{o} \rightarrow \hat{\mathbf{v}}^{2}$, see Fig. 1(b). The phases of such paths are $\xi\left(\overrightarrow{\mathbf{x}}^{j}\right)=\exp \left(i k\left(\left(\hat{\mathbf{i}}^{j} \cdot \mathbf{o}\right)-\left(\hat{\mathbf{v}}^{j} \cdot \mathbf{o}\right)\right)\right.$ and the phase product they contribute can be expressed as:

$$
\left.c_{\hat{\mathbf{v}}^{1}, \hat{\mathbf{v}}^{2}}^{\hat{\mathbf{i}}^{1} \hat{\mathbf{x}}^{2}}, \overrightarrow{\mathbf{x}}^{1}\right) \propto e^{i k(\boldsymbol{\omega} \cdot \mathbf{o})} \quad \text { with: } \quad \boldsymbol{\omega}=\hat{\mathbf{i}}^{1}-\hat{\mathbf{i}}^{2}-\left(\hat{\mathbf{v}}^{1}-\hat{\mathbf{v}}^{2}\right) .
$$

We suggest to approximate the covariance as the integral of Eq. (3) over all positions of the scatterer o in the $3 \mathrm{D}$ volume. In [3] we compute this integral analytically assuming the material slab has infinite extent in the $x y$ dimensions and thickness $L$ along the $z$ axis. The integral is non zero only when $\boldsymbol{\omega}=0$, leading to memory-effect type pairs, where the displacement between the illumination directions is equivalent to the displacement between the viewing directions, which we denote using $\hat{\Delta} \equiv \hat{\mathbf{i}}_{x y}^{2}-\hat{\mathbf{i}}_{x y}^{1}=\hat{\mathbf{v}}_{x y}^{2}-\hat{\mathbf{v}}_{x y}^{1}$, where $\hat{\mathbf{i}}_{x y}, \hat{\mathbf{v}}_{x y}$ denote the $x y$ coordinates of the corresponding 3D vectors. For such directions we also denote the angle between the illumination and viewing vectors using $\hat{\tau} \equiv \hat{\mathbf{i}}_{x y}^{1}-\hat{\mathbf{v}}_{x y}^{1}=\hat{\mathbf{i}}_{x y}^{2}-\hat{\mathbf{v}}_{x y}^{2}$. We denote by $\rho(\hat{\tau})$ the phase function corresponding to the portion of 


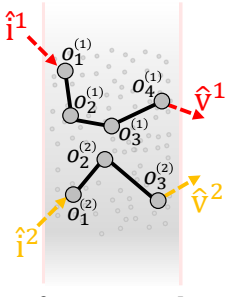

(a)

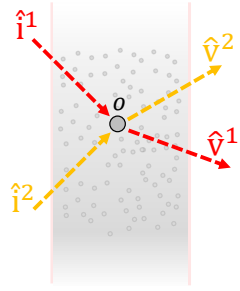

(b)

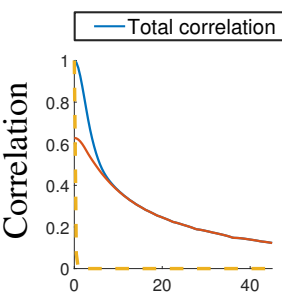

$|\hat{\boldsymbol{\Delta}}|[\mathrm{deg}]$

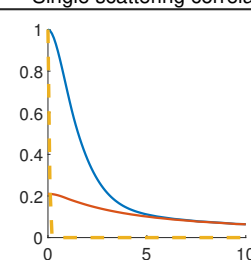

$|\hat{\boldsymbol{\Delta}}|[\mathrm{deg}]$

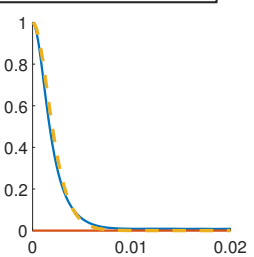

$|\hat{\boldsymbol{\Delta}}|[\mathrm{deg}]$

(e) $O D=100$

Fig. 1: Memory effect models: [Left] Path integral models for speckle correlations. [2] expresses correlation as an integral over all pairs of paths through the volume (a), while we suggest to approximate it by considering only pairs of paths that scatter at a single scatterer (b). [Right] We compare the diffusion theory [4] model for the ME with the exact prediction of the Monte-Carlo simulator [2], and our single scattering approximation. The different subfigs visualize volumes of three different thicknesses corresponding to different optical densities. We use scattering parameters representing tissue, in particular a narrow forward scattering phase function with $g=0.97$. The single scattering approximation is invalid at low angles $\hat{\Delta}$ where intensity dominates, but for larger ones it explains all correlation even for material thickness of $O D=3$ where light paths scatter more than once. This suggests that multiple scattering paths decorrelate quickly and do not contribute to the correlation. In contrast, due to the narrow phase function, the diffusion model becomes accurate only for very high optical depths as visualized in (e).

energy that a single scatterer illuminated by incoming direction $\hat{\mathbf{i}}$ scatters toward viewing direction $\hat{\mathbf{v}}$, and by $\sigma_{t}, \sigma_{s}$ the extinction and scattering coefficients. With this notation the single scattering correlation integrates to [3]:

$$
\mathcal{C}(\hat{\tau}, \hat{\boldsymbol{\Delta}})=L \rho(\hat{\tau}) \sigma_{s} e^{-\sigma_{t} L} e^{i k \frac{L}{2}(\hat{\boldsymbol{\Delta}} \cdot \hat{\tau})} \operatorname{sinc}\left(\frac{k L}{2}(\hat{\boldsymbol{\Delta}} \cdot \hat{\tau})\right) .
$$

Evaluation: In Fig. 1(c-e) we evaluate this result as a function of illuminator displacement $\hat{\Delta}$, considering a few material thicknesses. We compare the single scattering approximation of Eq. (4) with the full MC covariance of [2], which we treat as exact, as it was exhaustively compared against exact wave optics solvers. We see that as the illuminator displacement $\hat{\boldsymbol{\Delta}}$ increases, the single scattering approximation becomes accurate. The single scattering approximation is exact even for material of optical depth 3 , where most light paths scattered much more than once. Yet, light paths which scattered multiple times decorrelate quickly and they do not contribute much to the correlation. For very large optical depths the single scattering approximation becomes exponentially low and is inaccurate. At the same time, most practical applications of the ME are relevant at low to medium ODs [1], as for high ones the correlation decays to zero at very small angles (notice the different $\mathrm{X}$-axis ranges in Fig. 1(c-e)). Fig. 1 also evaluates the diffusion approximation to speckle correlation [4]. This approximation is valid only for very thick material where correlation is rather low, and is way too pessimistic for low to medium thicknesses.

Discussion: In [3] we show that the closed-form expression for the single scattering correlation Eq. (4) improves our understanding of speckle statistics, providing simple explanations for many previously reported properties, and also allows us to discover previously unreported properties. We hope this understanding will boost the design of new speckle based computational imaging systems. In particular, speckle correlation serves as a gating mechanism which provides direct access to the single scattering component and the phase function of the material. This offers a lot of potential for material acquisition applications where one is interested in measuring the properties of the material from its scattering profile.

Acknowladgments: This work was supported by European Research Council Horizon 2020 635537, ISF 1947, NSF-BSF award 2008123/2019758 and NSF Expeditions award 1730147

\section{References}

1. M. Alterman, C. Bar, I. Gkioulekas, and A. Levin. Imaging with local speckle intensity correlations: theory and practice. ACM TOG, 2021.

2. C. Bar, M. Alterman, I. Gkioulekas, and A. Levin. A monte carlo framework for rendering speckle statistics in scattering media. ACM TOG, 2019.

3. C. Bar, M. Alterman, I. Gkioulekas, and A. Levin. Single scattering modeling of speckle correlation. In ICCP, 2021.

4. S. Feng, C. Kane, P. A. Lee, and A. D. Stone. Correlations and fluctuations of coherent wave transmission through disordered media. Phys. Rev. Lett., 1988. 